\title{
Accelerometer-based on-body sensor localization for health and medical monitoring applications
}

\author{
Alireza Vahdatpour ${ }^{1,2}$, Navid Amini ${ }^{1,2}$, Wenyao $\mathrm{Xu}^{1,2}$, and Majid Sarrafzadeh ${ }^{1,2}$ \\ ${ }^{1}$ Computer Science Department, University of California, Los Angeles \\ ${ }^{2}$ Wireless Health Institute, University of California, Los Angeles
}

\begin{abstract}
In this paper, we present a technique to recognize the position of sensors on the human body. Automatic on-body device localization ensures correctness and accuracy of measurements in health and medical monitoring systems. In addition, it provides opportunities to improve the performance and usability of ubiquitous devices. Our technique uses accelerometers to capture motion data to estimate the location of the device on the user's body, using mixed supervised and unsupervised time series analysis methods. We have evaluated our technique with extensive experiments on 25 subjects. On average, our technique achieves $89 \%$ accuracy in estimating the location of devices on the body. In order to study the feasibility of classification of left limbs from right limbs (e.g., left arm vs. right arm), we performed analysis, based of which no meaningful classification was observed. Personalized ultraviolet monitoring and wireless transmission power control comprise two immediate applications of our on-body device localization approach. Such applications, along with their corresponding feasibility studies, are discussed.
\end{abstract}

\section{Keywords}

On-body device localization; Unsupervised activity discovery; Motion analysis; Ultraviolet monitoring; Transmission power control

\section{Introduction}

Wearable embedded system technology has resulted in utilization of body-wearable sensor networks for remote health and activity monitoring. Therefore, body-wearable sensor networks enable a personalized approach to health and wellness. Such networks will provide promising applications in medical systems to enhance people's quality of life, to facilitate independent living, and even to save the lives of people bearing the risk of sudden attacks (e.g., cardiac failure).

Pedometers and smart phones with motion sensors are among devices that are used to continuously monitor life characteristics such as the daily activity and exercise. With the production of ultra low power sensor interfaces such as Sensium [5], it is expected that smart band-aids become widely used for health and medical monitoring applications. Smart bandaids can be used for monitoring several metrics such as the daily energy expenditure, body

\footnotetext{
(C) 2011 Elsevier B.V. All rights reserved.
}

Publisher's Disclaimer: This is a PDF file of an unedited manuscript that has been accepted for publication. As a service to our customers we are providing this early version of the manuscript. The manuscript will undergo copyediting, typesetting, and review of the resulting proof before it is published in its final citable form. Please note that during the production process errors may be discovered which could affect the content, and all legal disclaimers that apply to the journal pertain. 
temperature, skin moisture, heart rate, and other human vital signs. The proliferation of these wearable devices results in higher diversity of their usage. In addition, being non-intrusive in daily activities requires these devices to be adaptable to individual users' habits.

Continuous monitoring of the human body is becoming pervasive with the advent of wearable devices capable of processing and storing large of amounts of an individual's health data (e.g., FitBit [2] and BodyBugg [1]). A number of systems make a connection between mobile sensing devices and existing home appliances; [13] aims at increasing the user's activity levels by disabling the TV remote unless enough activity points have been recorded through the on-body accelerometers.

Due to of the physiological requirements, or comfort, smart band-aids are attached to different locations on the body. The problem of misplaced/displaced sensors has not been given enough consideration in the pervasive computing society. Since we either exactly know the current location of sensors or the location of the sensor is not inherently required, we merely look into the data. Nevertheless, the effectiveness of heath monitoring devices is heavily contingent on the correctness of sensor installation/placement. As such, more work is needed to overcome several limiting challenges for optimal validity of data. Ignoring these issues will result in faulty interfaces, responsible for unfair physical activity gaming or false physical activity recording.

There are many technologies that enable movements capturing; among them, accelerometers are mostly quoted by researchers, since they are already present in several devices, have a low power consumption and a reasonable price, and yield excellent performances [23, 25, 34, 20]. Surprisingly, accelerometers have also been deployed by biologists on animals (rats [33] and cheetahs [14]) to study their movement model, as well as to identify their behavioral patterns.

There are at least four reasons why it is necessary or beneficial to automatically discover the location and the placement of these devices on the user's body.

1. Correctness: Wearable non-intrusive embedded systems are used to remotely and ubiquitously monitor subjects conditions such as basal body temperature, skin moisture, and ultraviolet exposure. Correct functionality of these monitoring devices highly depends on the correct placement of them on the subject's body. For example, Table 1 summarizes the normal temperature range variation for normal adults according to the location of the thermometer on the body [18]:

It is clear that the temperature varies in different body areas. If in each use a user attaches his temperature monitoring device to different regions on the body, clinicians and care-givers will be misguided by the data variation. Therefore, it is essential for the clinicians to know the placement of the sensing systems on the body, so that the data collected from the user becomes valuable and its analysis results in correct hypotheses.

2. Accuracy: The operation of motion and energy expenditure monitoring systems heavily relies on the placement and orientation of motion sensors, which usually include accelerometers and gyros. The functionality of pedometers is an example of the relation between the accuracy and the device placement. The step counting accuracy changes if the pedometer is attached to anywhere other than the waist, because the user's movements will be projected differently on the accelerometer. In an observation, we attached 6 pedometers to different regions on a subject body and asked him to take 220 steps. Table 2 shows how the location of the device greatly impacts the number of steps counted by each pedometer: 
It is clear that in a location-aware pedometer, a simple dynamic threshold control technique can dramatically enhance the accuracy of the step counting. Energy expenditure estimation via motion sensing [32] is another application where the outcome accuracy highly depends on the accurate classification of the type of the movement performed by the user (e.g., walking, jogging, running, jumping, etc.) and the placement of the accelerometer on the body [13]. If the user wears an accelerometer on his/her foot instead of the waist, the accuracy of the activity detection degrades dramatically. This is because the activity detection models implemented in the device are tuned for waist motions. As a result of wrong activity detection, the system will overestimate or underestimate the user's caloric expenditure.

3. Communication Optimization: Intrabody communication (IBC) is a different wireless communications technology that uses human's body as the transmission medium. This technology is grouped into two categories: waveguide IBC and electric-field IBC. Participation of the body as a transmission channel will force future bodywearable sensor networks to heavily rely on human-body channel measurements, where the surrounding environmental effect is imperceptible comparing to the effect due to the human body. Lately, investigators have substantiated significant variations of the transmission attenuation at different locations of the body for waveguide IBC [44] and electric-field IBC [47].

Furthermore, it is known that the human body deteriorates the performance of radio transceivers. Not only does such deterioration impact in-body communications, but, in fact, it noticeably degrades the performance of off-body and on-body communication systems [40] and [36], as well. Automatic on-body localization enables wireless devices to control the communication power according to the anticipated channel quality so as to compensate for the negative impact of the body.

4. Usability and Adaptability: Pervasive use of monitoring systems requires enabling the users to change sensors location and placement according to their life style, clothing, and activities. Not only does the on-body device localization enhance the usability of these devices, but also it provides the opportunity for software developers to design smarter devices, which can automatically adapt to users and their environment. Automatic device localization allows the smart phones to rapidly and autonomously adapt to users needs. Different placements of the device on the body can be interpreted as the users desire for an application or a major change in the environment. For example, as soon as the user mounts the smart phone on her upper arm, the smart phone should activate its gym/music mode.

This paper, builds on the work in [42] by taking an end-to-end view of our proposed on-body sensor localization technique. Our technique automatically discovers the on-body location of medical monitoring devices using acceleration data. Accelerometer is one of the most widely used types of motion sensors, which is used for a variety of applications such as device orientation detection, game controlling, shock protection, and activity discovery. Given that accelerometers are relatively cheap and consume low energy, nowadays they are embedded in most of the high-end portable systems. Our developed technique allows both on-line and offline discovery of the device location on the body. Our technique consists of two main phases: unsupervised activity discovery and supervised location estimation. We investigate two applications of our location discovery approach in body-wearable sensor networks. In the first application, a personalized ultraviolet monitor is envisioned to use the inferred location to improve its accuracy, i.e., to differentiate one body location from another and make more informed recommendations, accordingly. The second application utilizes the discovered location to provide more energy-efficient on-body wireless communications without sacrificing the reliability; data transfer, at unnecessarily high transmission power levels, not 
only diminishes the lifetime of the body-wearable sensor networks, but also incurs excessive interference.

In the remaining parts of the paper, we first review the related work. Afterwards, the description of the technique is presented. In the next section, we detail the experimental results followed by the aforementioned feasibility studies to justify that on-body sensor localization is worth implementing for such applications. Finally, the last section provides the concluding remarks.

\section{Related Work}

The problem of localization on the human body has been addressed by only a few number of researchers before. The most well known study is conducted by Kunze in [28] and [29]. While these studies address the problem of localizing devices on the body, the results of their classification is limited to four exact locations (wrist, breast pocket, trousers pocket, and right eye). As denoted by the authors, the locations that have been chosen represent typical location of appliances and accessories. In contrast, our approach is designed to discover device location on any region on the body. As a result, not is it only applicable in accessories and wearable systems (with higher diversity in discovering the location), but also it can be leveraged in smart band-aids and electronic implants.

A number of studies in mobile and smart phone area have also addressed simplistic device localization problem, where, for example, it is desired to detect whether a cellphone is on the table or in the pocket [22].

Accelerometer data has also been used in other studies to improve the utility of portable devices. For example, the study by Lester et al. [31] uses motion data to determine if two portable devices are carried by the same person.

Supervised techniques for activity detection using on-body sensors have been studied widely in the last decade [11]. All of these studies leverage body-fixed sensors and predefined models for activities. In the recent years, a few studies have addressed unsupervised classification of human activities [37, 41, 43]. We use the unsupervised most frequent activity detection technique proposed in [43] as part of our approach for on-body device localization.

\section{Overall Approach}

The essential idea behind the on-body localization is to analyze the acceleration data when the user is performing a specific activity. On the other hand, requiring the subjects to perform a specific activity will degrade the practicality of the solution and makes it user dependent. The study by Welker et al. [46] shows that on average people take 8265 steps per day (performing only normal daily activities). Such a large number of the steps clearly shows that walking is the most frequent and consistent activity people perform throughout the day (excluding non active states such as sleeping and sitting). Hence, the walking activity could be used as an identifier for our device localization technique. However, since the location, placement, and orientation of the accelerometer on the body is not known a priori (and may change in each use), the projection of the body movement patterns on the sensor is not predictable. Hence, it is impossible to define a generic model for detecting the walking activity which is valid for all placements and locations of the accelerometer. Because of the same reason, all of the studies in activity detection assume that the location of the accelerometer is known and is restricted to one or a few places. In this study, we use an unsupervised activity discovery method to discover time intervals that include walking patterns in long time series data. An important advantage of unsupervised techniques is that these methods derive the activity models during the run-time and, hence, they do not require predefined activity models and patterns in the setup phase [41]. 
Our unsupervised technique discovers walking activity occurrences based on their frequency and consistency during long time intervals. Once the time series subsequences representing walking activity are discovered, they are analyzed with a supervised classification technique. We use a support vector machine (SVM) to estimate the location of the device based on the frequency and time domain properties of the time series subsequences that represent the walking activity. In the following, we discuss each phase of the technique separately.

\subsection{Most frequent activity discovery}

Discovering most frequent activities has been in the interest of the research community in the recent years. Motif detection in time series data is a technique to discover the frequent subsequences in a longer time series [16]. A recent study by us [41] has extended the motif discovery mechanism to multi-dimensional time series data for discovering the most frequent activities in wearable systems. However, as the study by Chiu et al. [16] suggests, motif discovery is computationally intensive and is not suitable for embedded computing and applications with a huge amount of data. Hence, in this paper, we use an approach similar to the method presented in [43] for discovering the most frequent activity (walking) in 3dimensional accelerometer data. Our approach for most frequent activity discovery consists of two steps. In the first step, each dimension of the accelerometer data is analyzed separately to discover frequent patterns. In contrast to [41], we use a binning approach to classify similar patterns in single dimensional accelerometer data. In the second step, classified patterns are grouped together with a clustering approach to form the activities. Next, we describe each step of the frequent activity discovery method:

3.1.1. Activity primitive discovery in single-dimensional time series-Numerous approaches have been used to discover similar patterns in a time series data. Although using motif discovery results in accurate classification of patterns, it is computationally intensive and sensitive to noise in the data. Here, we classify patterns in single dimensional accelerometer data based on the total movement that is resulted from the activity in the corresponding axis. We define an activity primitive to be a subsequence between two consecutive stable regions (or default sensor value) in the accelerometer time series data. Such subsequences are mostly observed as bell shape patterns (with local minimum or maximum points) or linear slopes between two stable values in human actions, which depending on the speed and nature of the activity have different length and frequency. The acceleration data can be converted to displacement information, using the following equation on a subsequence of length $m$ $\left(a_{1} a_{2} . . a_{m}\right)$.

$$
\text { displacement }=D \cdot \sum_{k=1}^{m} \sum_{i=1}^{k}\left|a_{i}-a_{1}\right|
$$

where $D$ is the calibration constant factor, which depends on the placement of the sensor and other parameters such as the frequency of data sampling. Although the location of the sensor in not known in our application, since displacement information is used to compare patterns in the same accelerometer, it is constant for all patterns and can be eliminated from the calculations. Figure 1.a depicts three accelerometers and several activity primitives classified in them (labeled by alphabets).

Algorithm 1 presents the method for extracting primitives of activity subsequences projected on single-dimensional time series. The algorithm extracts the patterns from the time series, classifies them based on the physical attribute of the data and according to the discretization cardinality, and assigns a symbol to them. It also assigns a starting time to each symbol. There are a variety of methods in literature regarding effective discretization, especially in time series. 
Here, in order to discretize the calculated displacement and assign symbols to it, we consider using the probability distribution of the calculated displacements and assigning symbols to primitives such that primitives are classified fairly considering the variation of displacement in the application runtime.

Note that in contrast to motif discovery method used in [41] for detecting recurrent patterns in time series, this algorithm leverages domain specific attributes of data (here, being the motion sensing data, which is used frequently in wearable systems). Regardless of the method used to discover recurrent activity patterns in the single-dimensional time series, the output of this phase is passed to the multi-dimensional activity and abnormality detection algorithm, which is described in the next section.

Algorithm 1 Single-dimensional activity primitive extraction

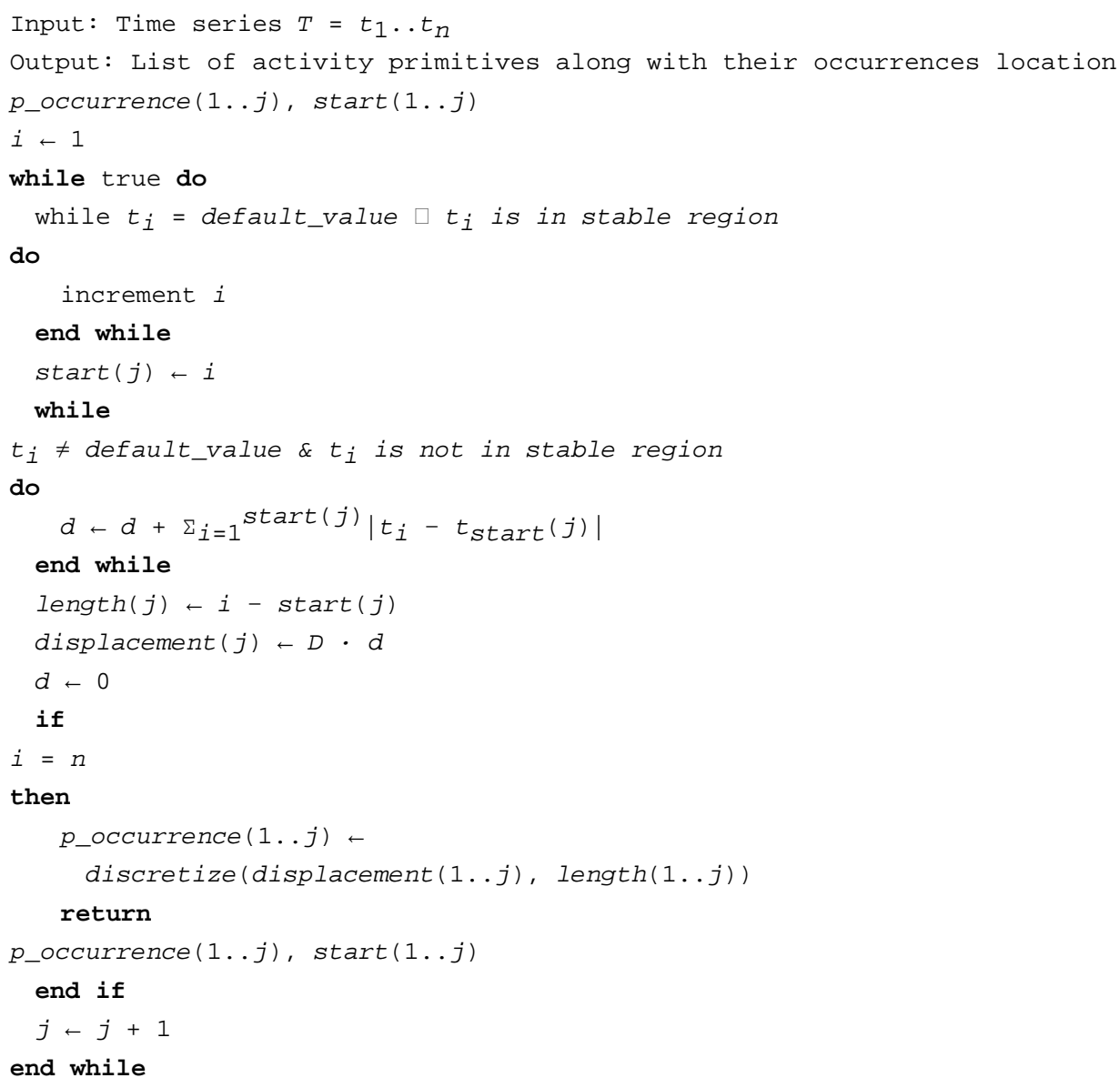

3.1.2. Activity in multi-dimensional time series-By using 3-dimensional accelerometers, activity motions are projected on one, two, or three dimensions of the accelerometer. Since the original orientation and placement of the accelerometer is unknown, all dimensions should be considered together in order to discover recurrent activity. If only a subset of dimensions is used for counting the occurrence of activities, activities with similar projection will be classified together and over counted. As a result, we first use a graph clustering approach to construct activity structures in multi-dimensional data. This step 
basically groups several activity primitives from different dimensions together according to their temporal characteristics.

In order to construct the multi-dimensional activity structures, we first convert the list of discovered activity primitives into a weighted directed graph, so that a graph clustering mechanism can be applied to construct the model of activities in multi-dimensional data. In the proposed graph, each vertex represents an activity primitive, and the weight on the vertices represents the number of occurrences of the primitive in the corresponding time series. The weight on each directed edge in the graph is calculated by the following equation:

$$
e(i, j)=\frac{\text { coincidence }(i, j)}{\text { total_occurrences }(j)}
$$

The coincidence $(i, j)$ denotes the number of times there is temporal overlap between occurrences of primitives $i$ and $j$. As a result, $e(i, j)$ will be at most 1 , when all the occurrences of primitive $j$ have overlap with occurrences of primitive $i$, and it is at least 0 , when there is no overlap between occurrences of primitives $i$ and $j$.

Afterwards, a graph clustering approach is used to construct multi-dimensional activities structure from the primitive coincidence graph. Algorithm 2 presents our method for constructing the activity structures from the coincidence graph. This method is similar to the clustering mechanisms proposed in [10]. Clustering the primitives starts with sorting the list of vertices based on the number of occurrences of the primitives. Then, the most frequent primitive is selected as a candidate core for activity structure, and the graph is searched for primitives with high occurrence correlation with the candidate core primitive. If there are several primitives with equal frequency, the one with the larger number of highly correlated neighbors is selected as the core primitive. The threshold for the correlation is set to $1-\beta$, where $\beta$ is the abnormality frequency constant. Upon construction of an activity by clustering correlated primitives, selected primitives are removed from the graph, and the algorithm continues for discovering new activity structures by selecting a new core activity primitive.

Figure 1.c illustrates the results of applying the algorithm to the coincidence graph in Figure 1.b (the weights in the graph are omitted to make the figure readable). As it is denoted in the figure, the activity structure extracted contains primitives $\mathrm{E}, \mathrm{D}, \mathrm{G}, \mathrm{O}$, and $\mathrm{N}$ ( $\mathrm{G}$ is the activity core primitive, due to its frequent number of occurrences). Note that the effective construction of activity structures highly depends on the frequency of primitives, and in a real world application the frequency and coincidences are significantly higher $(\beta$ will be set to less than $0: 1$ in real world applications). The depicted graph is formed from a set of short time series and is selected to be small to better illustrate the logic behind the algorithm.

Algorithm 2 Activity structure construction from the primitives coincidence graph

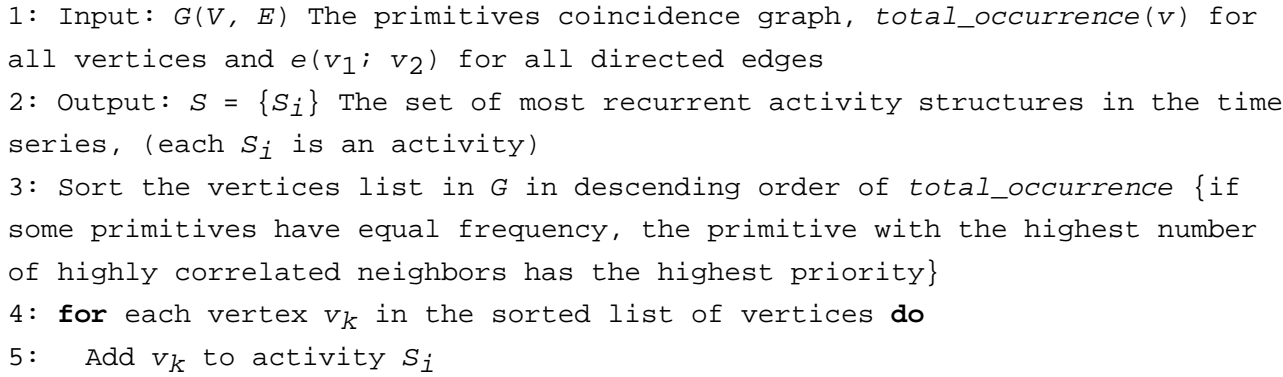


6: for all neighbors of $v_{k}$, if $e_{(j, k)}>1-\beta$

do

7: Add $v_{j}$ to activity $S_{i}$

8: remove $v_{j}$ from the graph

9: end for

10: $i \leftarrow i+1$

11: Update the sorted list of vertices

12: end for

13: return

$S=\left\{S_{1}, \ldots, S_{i-1}\right\}$

For detailed description and evaluation of unsupervised multi-dimensional activity detection algorithm, we refer the reader to [41]. However, it should be noted here that the execution overhead of this method is linearly correlated to the execution overhead of single dimensional motif discovery algorithm. The computational intensity of algorithms for motif discovery can be reduced by introducing assumptions about the input signals such as the minimum and maximum length of subsequences and the frequency of activities.

\subsection{Location discovery via SVM}

Once the walking interval is discovered via the unsupervised method, the walking subsequence is analyzed to discover the location of the accelerometer on the body. We extract several frequency and time domain features from the walking subsequences. Frequency domain features are analyzed to evaluate the impact of each step on the accelerometer. In each step, the energy of the impact between the foot and the floor is distributed in the body. The closer the sensor is to the foot (the origin of the strike in each step), the stronger is the energy sensed by the accelerometer. Time domain features are evaluated to analyze the motion range of the sensor in all three directions (we are using tri-axial accelerometer). It is clear that some limbs (e.g., forearms) move more freely than other limbs (e.g., chest).

In addition to analyzing the walking patterns, the time series subsequences representing general activity (non-walking) were also analyzed. In this regard, we designed an algorithm to count the number of times the orientation of the device (body part) changes over time. The algorithm first approximates the orientation of the sensor in each 10 second interval, via calculating the DC value of the Fourier transform. Then, the total number of orientation variations is calculated for a long time series by comparing the consecutive approximate orientations. Table 3 and Figure 2 summarize six features extracted from walking and non-walking subsequences and the intuition behind using them for location classification. Figure 2.a depicts a time series representing multiple walking steps in a 3 second interval (captured on the forearm). The power spectrum depicted in Figure 2.b is the sum of the frequency power spectrum of the 3dimensions. As illustrated, feature $\mathrm{A}$ is the maximum amplitude among all the dimensions.

$\frac{A}{C}$ and $\frac{A}{B}$ are the ratio of the maximum amplitudes in different axes. $\mathrm{D}$ and $\mathrm{F}$ represent the maximum energy (which is at $1.85 \mathrm{~Hz}$ ) and the overall energy captured by the accelerometer, respectively. Note that although Table 3 consists of merely 6 features, our SVM uses 17 features, which also includes variations of those 6 features.

It is noteworthy that for each placement of a sensor on the body, the relation between coordinate systems of the sensor and the subject's body varies. Hence, no assumption can be made during the feature extraction regarding the initial orientation of the sensor. As shown in Table 3, these features either represent an accumulation value calculated over all the directions or depict the maximum of a property among the three dimensions. 
Figure 3 illustrates the variation of features A (maximum amplitude), D (maximum energy), and $\mathrm{F}$ (sum of the energy) for a subject, when the accelerometer is mounted on different regions on his body. While as a result of strides variation, the features span over the normalized y axis, it is clear that different body locations exhibit different ranges for each feature. In the next section, we evaluate the performance of SVM in classifying the location of the sensors into six different regions depicted in Figure 5. Each of these regions has semi-independent movements during human activities. We performed several experiments to evaluate the effect of dividing each of these six regions into smaller areas. However, due to the motion characteristics of the human body, no meaningful statistical difference was observed between the features of the detailed areas. Hence, we conducted the rest of our experiments based on these six areas on the body. In the next section, we present the experimental evaluation of our classification technique for the six regions depicted in Figure 5.

\section{Experimental Results}

We used the acceleration logging device from Gulf Coast Data Concepts to conduct our experiments [3]. The $\pm 6 g$ gain tri-axial accelerometer used in the device, does not saturate during normal activities. We set the sampling rate to $160 \mathrm{~Hz}$. The device is suitable for long intervals of data collection because it has 1 GB of memory. In addition, the size of the device is relatively small $(1 \mathrm{in} \times 3.5 \mathrm{in} \times$. $75 \mathrm{in})$ and does not interfere with the normal activities of the subjects.

25 subjects (20 males, age between 18 and 35) participated in our study; each subject wore 10 sensors on 10 different parts of his/her body, according to the sample setup shown in Figure 5. No instruction was given to the users regarding the exact placement and the orientation of the sensors and users were allowed to place the devices anywhere in the requested body areas. Subjects were allowed to choose to attach the sensors to the skin or to put them on their clothes. Each subject had sensors on for 30 minutes, during which he/she was asked to perform a sample set of his/her own daily activities in the university environment. We required the users to include a number of walking intervals in their activities. The collected time series data was divided into 3 minute subsequences. The total of 2500 time series samples were collected in this phase. We used an open source SVM Library (LIBSVM [4]) for implementation of the classifier. A radial basis function (RBF) was used for the SVM kernel function. Parameter selection and tuning of of the kernel function was done via cross-validation and grid-search techniques.

\section{Walking interval discovery}

For each sample, the walking intervals were discovered via the unsupervised technique for the most frequent activity detection. $96 \%$ of the time series samples were detected to include walking periods. This rate is high due to the fact that subjects were asked to include walking periods in their activity. The walking discovery algorithm had a consistent accuracy in detecting walking occurrences in the time series. On average $93 \%$ of all walking strides were discovered and classified correctly (versus $84 \%$ reported by [29]). In discovering the walking occurrences, the highest accuracy was in the foot area with 95\% accuracy and the lowest was in hand area $(89 \%)$ due to the high variation in the motion patterns. Figure 4 depicts an example of data collected from a sensor placed on a subject's arm. On average, a $2 \%$ false positive rate was observed, which denotes the number of non-walking activity occurrences that were labeled as walking (compared to a $4 \%$ rate reported by [29]). Since we were interested in finding relatively longer walking time intervals, the effect of false positives was eliminated as these occurrences were sparsely distributed in the time series data. 


\section{Sensor location detection}

In the first experiment, we trained and tested the localization SVM for each participant separately. Of the 100 time series samples collected from each subject, 20 samples were used for training and the remaining 80 for the testing. As expected, the classification result was near perfect, since the motion characteristics for each subject is consistent during his/her activity. The minimum, mean, and maximum overall classification precision for 25 subjects were $88 \%$, $94 \%$, and $100 \%$, respectively. However, having training data from each user is not a valid assumption in real life scenarios, where the algorithm is supposed to work on off-the-shelf devices for unknown users. Hence in the second experiment, we trained the system using 500 randomly selected samples out of 2500 time series. The average classification accuracy in this scenario was $89 \%$. Table 5 presents the classification precision and recall rate for each region separately. Note that direct comparison to the results of sensor localization in [29] is not possible since the set of locations in [29] is more limited and different from ours. However, the average classification accuracy reported by authors in [29] is in the same range reported here $(\sim 90 \%)$ and confirms the applicability of localization methods in improving the utilization of wearable (our study) or portable devices ([29]).

Table 4 presents the details of false classifications for each area on the body. Head has the least classification accuracy, because it generally mimics the motion characteristics of the upper extremities. Classification of the shin area is performed perfectly, since the impact of each step on the shin area is significantly higher than other locations.

Finally, we performed analysis to study the feasibility of classification of left limbs from right limbs. No meaningful classification was observed using the SVM. This is due to the fact that the sensors were mounted on different sides of the limbs by different users, and hence the accelerometer coordinate system changed in each experiment.

In an attempt to find asymmetries between left limbs and right limbs, among the six investigated areas on the body, we decided to quantify the mobility of the upper arms during the walking activity. Once the walking interval is discovered by our unsupervised method, we extract a sequence of 100 gait cycles. In order to detect starting indices of each gait cycle, for each sample point in the combined acceleration signal, we find the length of the longest interval in which the sample point has the largest value comparing to its subsequent points. We discovered that the start point of each gait cycle remains maximum for a relatively long time, although it might not be the global maximum of the period. By means of this idea, the starting indices of each gait were found in an accurate fashion. We applied this algorithm to the combined timenormalized accelerometer signal, i.e., the square root of the sum of the squared acceleration components along the $\mathrm{x}, \mathrm{y}$ and $\mathrm{z}$ axes produced by the sensors attached to the left and right upper arms, in order to detect cycles of gait.

For each gait cycle, arm's activity based on $\mathrm{x}, \mathrm{y}$ and $\mathrm{z}$ accelerations were calculated and compared for both sides; In order to estimate arm usage, arm's activity was compared to a defined threshold. If the activity is smaller than a threshold, the arm is considered as motionless; otherwise, it is considered as 'in use'. If the difference between the left arm's activity and the right arm's activity is positive and the latter is larger than the threshold, usage is classified as a left arm usage. If such difference is negative and the right arm's activity is greater than the threshold, the usage is classified as a right arm usage. The flowchart in Figure 6 provides an overview of the asymmetry-detection process.

To define the threshold, we extract an episode of rest data, e.g., when the user does not move his/her arms. The mean value for activity during the rest for left and right arm is used to define the threshold. Also, it should be noted that the starting indices of each gait cycle can be found 
by employing many different methods, such as locating the zeros of the signal and computing the signal energy.

The cycles where activity is greater than the threshold, are estimated as a percentage of the total cycles and were defined as usage percentage. Table 6 presents the percentage of the left arm usage and right arm usage in 100 gait cycles. During walking, the arms show a cyclic movement, where the right and the left arms are used equally (almost 50-50\%). Although both arms were, on average, used equally during walking, it should not be inferred that this is the case in each gait cycle. For example, external work (carrying a load) may impact this similarity.

In conclusion, an activity like walking provides unique measurements all over the user's body, except for left-right limbs.

\section{Applications and feasibility studies}

In light of the promising granularity of the proposed location discovery technique, a great deal of health monitoring applications can take advantage of this method. In fact, our technique will enable such applications to yield higher accuracy, achieve a longer battery lifetime and become adaptable to a certain degree, and even in some cases to function correctly. In this section, we introduce two active projects at the UCLA Wireless Health Institute benefitting from the proposed technique.

The fact that the human skin at different body sites is not equally sensitive to the ultraviolet radiation, suggested us to make the third prototype of our personalized ultraviolet monitor [8] adaptable to different body areas. In the context of body-wearable networks, recent studies show that the effect of human body is more influential than the surrounding environmental effect, especially when there is no line-of-sight path in the propagation channel. Transmission power control methods alleviate a large number of problems associated with extending the wireless system's battery life without compromising the reliability and data communication rate. Hence, in order to build energy-efficient body-wearable medical monitoring devices, it is imperative to model the wireless channel for different parts of human body and adapt the transmission power, accordingly.

\subsection{Personalized Ultraviolet Monitoring}

Recently, our group has discovered an interesting application for wireless sensor technology, where we conceived, developed and tested two prototypes of a wireless personalized ultraviolet monitoring device [8]. The device is able to measure and monitor the ultraviolet radiation in a medically meaningful way.

The worldwide escalating rate of skin cancer is attributed to significant increase in the amount of ultraviolet light that reaches the Earth's surface. In contrast to infrared radiation, which merely causes molecules within an object to vibrate, ultraviolet radiation possesses sufficient energy to damage the DNA carried by skin cells. Our personalized ultraviolet monitor is a band-aid-sized battery-operated device that can wirelessly communicate the UV index (a standard measure for the strength of ultraviolet radiation) data to the user's cell phone on which the software of the system runs. The system informs the users about their maximum recommended sun exposure time by calculating the UV index. Furthermore, given that the damage caused by ultraviolet radiation is accumulated over days, the system is able to keep a record of the amount of ultraviolet radiation received over a certain course of time, from a single day to a month. In our design, we have developed a model to determine the UV index with high accuracy. The maximum recommended sun exposure time is also derived by taking the user's skin type and sun protection factor (SPF) of the applied sunscreen into consideration. 
According to experimental studies, energy densities (total energy per unit area obtained by integrating irradiance with respect to time of exposure) in the range of $20 \mathrm{~J} / \mathrm{cm}^{2}$ to $100 \mathrm{~J} / \mathrm{cm}^{2}$ induce skin pigmentation and sunburn. In this regard, one of the key questions is to what extent the on-body location of the personalized ultraviolet monitor will matter.

Not coincidentally, the head, face and neck are where the majority of skin cancers occur due to the fact that such areas are frequently exposed to ultraviolet radiation [7]. Another main reason stems from the variations in the thickness of the skin on different parts of the body. The thicker the skin, the more layers of cells in them, and the longer it takes a sunburn to occur. It should be noted that multiple sunburns can further lead to skin cancer.

A number of investigators have sought various parameters for predicting and measuring ultraviolet sensitivity; various anatomical sites may afford different degrees of protection against the harmful effects of ultraviolet radiation. In one of such studies, investigators confirmed significant differences in erythema index at different anatomical sites. Erythema index represents the blood content of the inner layer of the skin caused by the pathological reddening of the skin, e.g., as a result of a sunburn. Using highly quantitative and reproducible methods, researchers in [39] found that the erythema index is quite variable, according to the anatomical body sites. In particular, they found that the chest and the thigh have the lowest erythema index, whereas the forehead, cheek, and neck are, by a factor of $2 \sim 3$, more sensitive to sunburn (see Figure 7 and Table 7).

The personalized ultraviolet monitor can easily exploit the proposed on-body device localization technique at the expense of adding a 3-d accelerometer. Accordingly, the third prototype will be able to, periodically and autonomously, adapt itself to the new body site to which it is attached. We anticipate that more conclusive interpretations will result from this new location-aware prototype, e.g., the risk of skin cancer as a function of body site among other factors and how differently various anatomical sites will respond to the ultraviolet radiation.

In order to find the solar exposure on different parts of the body, we conducted an experiment on two male subjects (heights $173 \mathrm{~cm}$ and $180 \mathrm{~cm}$ ), where we attached flat UV sensors of type ML8511 to fifteen different locations on their body. The experiment took place on the roof of an eight-story building on a typical sunny day in Los Angeles at an altitude of $300 \mathrm{~m}$ during the solar noon (the moment when the sun appears the highest in the sky). The subjects were asked to stand in an upright position with theirs arms hanging down by their sides. The two subjects, initially heading towards north, were asked to turn their body 90 degrees counterclockwise. We recorded the outputs of sensors for all four directions. As anticipated, solar UV exposure varies from one anatomical site to another. The horizontal sites on an upright person, including the shoulders, receive up to $70 \%$ of ambient UV radiation, while vertical sites receive noticeably less UV radiation. Table 8 compares the mean fraction of ambient UV radiation received at anatomical sites for our two subjects.

\subsection{Transmission Power Control for On-body Wireless Communications}

In another project, we target body-wearable sensor networks, in which the sensor nodes are strategically placed on the human body and wireless communications are conducted among nodes on the surface or in the vicinity of the body. We account for the impact of the human body on the wireless propagation channel and, accordingly, adjust the transmission power level to reduce the energy consumption of each individual sensor node. Examples of the systems benefiting from our power control methods are the personalized ultraviolet monitoring and the smart shoe systems. The former was introduced in Section 5.1 and the latter is intended to perform gait analysis and to assess the risk of fall. 
The human body includes liquid, bone and flesh, which, in a selective manner, absorb, reflect or scatter wireless signals. Hence, the nodes used in body-wearable sensor networks need to cope with lossy radio transmissions around the human body. Recent findings indicate that in the range 2-6 GHz, no energy can pass through the body. Rather, radio waves transmitted from an antenna diffract around the body and can reflect from arms and shoulders. Generally, attenuation rate and multipath effects increase at higher frequencies, therefore, a $2.4 \mathrm{GHz}$ signal weakens faster than a sub-GHz signal, e.g., 433, 868, and $915 \mathrm{MHz}$ carrier frequencies.

As part of the aforesaid project, we have studied the issues confronting $2.4 \mathrm{GHz}$ wireless communications around the human's body. Automatic on-body localization enables transmitters to control the communication power according to the anticipated channel quality. Human body causes large signal attenuation which has a remarkable effect on wireless link reliability. We conducted measurements so as to find spatial impacts on the correlation between transmission power and Receive Signal Strength Indicator (RSSI). RSSI is a useful link metric that represents the signal power averaged over 8 symbol periods of each incoming packet. We have evaluated the effect of human body by attaching transmitting sensor nodes to six different body positions namely, the head, upper arm (left and right), the forearm (left and right), the waist, the thigh (left and right), and the shin (left and right). The receiver node was taped to the subject's waist. It should be noted that the selection of body positions for this experiment is in accordance with the granularity of our device localization technique (refer to Figure 5).

We have used Digi's XBee RF Modules [6] to conduct our experiments. The XBee modules have been engineered to meet IEEE 802.15.4 standards and support the unique needs of low data rate, simple connectivity, and low-power sensor networks. They operate at the ISM 2.4 $\mathrm{GHz}$ frequency band, with a maximum nominal data rate of $250 \mathrm{kbps}$. Furthermore, XBee modules provide an RSSI measurement tagged to a specific packet. As for the transmission power, XBee modules can be configured to operate using five different transmission power levels ranging from 0 to 4 . The levels 0 to 4 , respectively correspond to power outputs of -10 $\mathrm{dBm},-6 \mathrm{dBm},-4 \mathrm{dBm},-2 \mathrm{dBm}$ and, $0 \mathrm{dBm}$.

Although a chip antenna demonstrates limited reliability and lower transmission range than a whip antenna, on account of its small form factor, we decided to use XBee modules equipped with chip antennas in both the transmitting and the receiving nodes. As a matter of fact, using a chip antenna has helped us to improve the repeatability and reproducibility of our experiments as well. With respect to radiation patterns, both antennas, albeit not perfectly, exhibit omnidirectional radiation patterns.

The experiments were repeated with three different pairs of nodes in the same environmental conditions to achieve statistical confidence. Measurements were performed on two male subjects on an open field to prevent radio wave reflections from the environment. The subjects were asked to fully extend their arms horizontally while performing the experiments pertaining to upper arms and forearms. In each experiment, the transmitting node sends 1000 packets at five different power levels and the recipient logs the packet's sequence number and the RSSI reading. More details regarding the experiment setup are listed in Table 9.

As shown in Figure 8, the average RSSI values gradually increase with the transmission power level. At the transmission power level of $-10 \mathrm{dBm}$, in experiments where we attached the transmitter nodes to the shin, head, and forearm, the resulting packet drop rate was dramatically high (more than 50\%). As a result of such a high packet drop rate, we inevitably report the RSSI value as $-100 \mathrm{dBm}$, which is lower than the receiver's sensitivity level. Expectedly, the experiment concerning the waist yields the best RSSI values, since the receiver is in the transmitter's line of sight. Indeed, the physical distance between the transmitter and the receiver 
in this case is only $10 \mathrm{~cm}$. This is why the data success ratio was always $100 \%$ even when the $\mathrm{XBee}$ modules operate at the lowest transmission power level.

On the basis of our experimental results as well as similar studies such as [27] and [24], RSSI values higher than $-80 \mathrm{dBm}$ are indicative of reliable communications. In fact, choosing such a conservative threshold of $-80 \mathrm{dBm}$ always allowed for communications with packet drop rates less than 5\%. Thereby, when we attach the nodes to the thigh, shin, and waist, as Figure 8 suggests, there is enough room to reduce the transmit power level without compromising the reliability of communications. For instance, transmission power reduction for data communications between the thigh and the waist will bring about up to $50 \%$ energy savings.

The shins and generally the lower part of legs turned out to be very favorable locations for onbody wireless communications. This is because, the legs are relatively close to the ground, where reflections increase the total received power with major contributions from the multipath components rather than the direct received signal, which in turn leads to less steep changes in the RSSI values. One of the most common applications for body-wearable sensor networks placed on the lower part of the human body is athlete's performance monitoring. Dealing with sensor displacement (and misplacement) in performance monitoring systems and generally, in on-body activity recognition systems has always been a matter of concern. In healthcare and medical applications, body-wearable sensor networks are expected to perform long-term monitoring, thus it is sensible for such systems to communicate their data at a lower transmission power. Performing transmission power control has several other advantages such as: reducing inter-network interference and thus improving the spatial reuse of wireless resources, increasing security as a packet with lower transmission power is not easy to intercept, and reducing the average contention at the MAC layer. Based on our experiments, the body location to which the node is attached significantly affects the radio propagation channel. The aforesaid advantages motivated the basic idea of integrating our on-body device localization technique into the process of dynamic transmission power control. To this end, we will derive different radio channel parameters and analyze them for different parts of the human body. Similar to the previous subsection, the energy consumption of the required accelerometer must be taken into consideration when estimating the battery lifetime of a certain body-wearable device.

\section{Conclusion and Future Work}

In the last few years, accelerometer-based entertainment and health monitoring applications have been receiving growing attention in the research and commercial communities. Surprisingly, delicate accelerometers are also being used to monitor animals, offering insight into a wide range of biological questions. However, the effect of accelerometer (and other sensors) placement on different parts of the body, despite its apparent significance, has received little consideration. While this error may be minimized through various techniques, additional error may occur as a result of sensor replacement.

We presented a technique for discovering the location of sensors on the human body, using acceleration data captured during daily activities. Our technique first leverages unsupervised activity discovery to detect time intervals, in which the user is walking. Then it uses a support vector machine (SVM) to analyze the patterns and estimate the position of the device on the body. We have presented extensive experimental results, which have been performed on a diverse set of participants. In our study, we were able to classify the shin area flawlessly, as the impact of each step on the shin is significantly higher than other locations. We also found that head has the least classification accuracy, since it generally mimics the motion characteristics of the upper extremities. Our experimental results demonstrate the significant difference that accelerometer placement can cause on the measurements used in ubiquitous 
entertainment and health applications. Ignoring these factors will lead to unfair physical activity gaming or erroneous medical monitoring. Despite our extensive experiments and corresponding analysis, there are still lots of open research problems worth further consideration.

Potential applications benefitting from our proposed on-body location discovery technique, namely ultraviolet monitoring and transmission power control for body-wearable sensor networks, were discussed and examined from the feasibility point of view. In the latter, we account for the impact of the human body on the wireless propagation channel and, accordingly, adjust the transmission power level to reduce the energy consumption of each individual sensor node. As part of the former project, we investigated the solar UV exposure on different parts of the body; we conducted an experiment on two male subjects and we found that more than $70 \%$ of ambient UV radiation is received by subjects' shoulders.

Recently, there exist dozens of ubiquitous mobile applications intended for tracking of daily physical activity. The problem with almost all these applications is that they do not take into consideration the dramatic effect that the accelerometer placement has on the measurement. It has shown that different users prefer to attach the sensors in different parts of their bodies in physical activity games that involve walking. The question is how different positions affect the accelerometer output and bias these games or other ubiquitous physical activity applications. Such bias should be taken into account in application design to enhance the user's experience. Furthermore, accelerometers give indirect and sometimes misleading indications of physical activity intensity. Unfortunately, many applications report only raw accelerometer values or simple derivatives, such as the number of steps. We are currently developing a set of applications for smart phones to enhance the adaptability and location sensitivity of these devices. In addition, we are investigating the use of acoustic sensors (to sense heartbeat sound) to improve the granularity of the location discovery.

More recently, scientists have utilized accelerometers to profile and identify users; for example, the use of gait analysis is less intrusive for implementing biometric authentications as opposed to other techniques such as voice, fingerprint or iris analysis. Kale et al. [26] and Gafurov et al. [21] used gait recognition to detect whether a device is being used by the owner. These biometrics and location-based approaches are complementary to our work. Likewise, Chang et al. [15] used accelerometers in television remote controls to identify individuals.

Gait related studies $[12,45,17]$ have shown that there are gender differences in human gait; females walk with lesser step width and more pelvic movement, while males move their shoulders more often. In our current experiments, in light of the gender imbalance among our participants, we were unable to articulate this problem. Modern gait analysis generally takes gender into account. A related study successfully examined gender recognition based on [12]. We anticipate that our future experiments, which involve accelerometer readings based on acceleration logging devices, should be able to detect differences in hip and shoulder movements, hence, identifying the sex of a user. A related study shows that accelerometer data while standing can to some extent describe how balanced the user walks [35].

We also proposed a method, which quantifies the arm's mobility during physical activity; our method provides insight to detect symmetry between the motions of right and left limbs. We plan to examine gait patterns to determine the presence of asymmetry and other alterations as they occur during running.

Our technique depends on the assumption that users are capable of walking, which may not hold for some users. It remains as future work to determine whether we can extend the technique for a person who is not able to walk. Studies have shown running gait can become different between left and right sides of the body. Asymmetry may not be distinguishable during initial 
stages of exercise, but it may arise with the development of muscle fatigue and/or changes in exercise intensity [30, 9]. As a matter of fact, the development of leg muscle fatigue [38] and changes in running vigorousness are known to alter running gait [19].

\section{References}

1. BodyBugg. http://www.bodybugg.com/ retrieved on June 2011

2. FitBit. http://www.tbit.com/retrieved on June 2011

3. Gulf coast data concepts. http://gcdataconcepts.com/x6-2.html/ retrieved on June 2011

4. LIBSVM. http://www.csie.ntu.edu.tw/ cjlin/libsvm/ retrieved on June 2011

5. Ultra low power sensor interface. http://www.toumaz.com/ retrieved on June 2011

6. Xbee modules. http://www.digi.com/products/wireless/point-multipoint/xbee-pro-series1-module.jsp

7. Your winter face. http://www.shincancer.org retrieved on 3/25/2011

8. Amini N, Matthews J, Dabiri F, Vahdatpour A, Noshadi H, Sarrafzadeh M. A wireless embedded device for personalized ultraviolet monitoring. BIODEVICES '09. 2009:200-205.

9. Arampatzis A, Brggemann G-P, Metzler V. The effect of speed on leg stiffness and joint kinetics in human running. Journal of Biomech. 1999; 12:1349C-1353.

10. Aslam, J.; Pelekhov, K.; Rus, D. SODA '99 Proceedings of the tenth annual ACM-SIAM symposium on Discrete algorithms. Philadelphia, PA, USA: Society for Industrial and Applied Mathematics; 1999. A practical clustering algorithm for static and dynamic information organization; p. 51-60.

11. Bao, L.; Intille, SS. PERVASIVE '04. Springer; 2004. Activity recognition from user-annotated acceleration data; p. 1-17.

12. Barclay C H, Cutting J, Kozlowski. Temporal and spatial factors in gait perception that influence gender recognition. Perception and Psychophysics. 1978; 23:145C-152. [PubMed: 643509]

13. Bouten CVC, et al. Effects of placement and orientation of body-fixed accelerometers on the assessment of energy expenditure during walking. Medical and Biological Engineering and Computing. 1997; 35:50-56. [PubMed: 9136191]

14. S ELC, H LG. Identification of animal movement patterns using tri-axial accelerometry. Endangered Species Research. 2008; 6:13C-23.

15. Chang K, Hightower J, Kveton B. Inferring identity using accelerometers in television remote controls. PerCom '09. 2009

16. Chiu B, Keogh E, Lonardi S. Probabilistic discovery of time series motifs. KDD '03. 2003:493-498.

17. Cho S, Park J, Kwon O. Gender differ-ences in three dimensional gait analysis data from 98 healthy korean adults. Clinical Biomechanics. 2004; 19:145-152. [PubMed: 14967577]

18. Crawford DC, et al. Which thermometer factors influencing best choice for intermittent clinical temperature assessment. Journal of Medical Engineering \& Technology. 2006; 30:199-211. [PubMed: 16864231]

19. Dutto D, Smith G. Changes in spring-mass characteristics during treadmill running to exhaustion. Med Sci Sport Exerc. 2002; 34:1324C-1331.

20. Fu L, Fellow I. Gesture stroke recognition using computer vision and linear accelerometer. 8th IEEE International Conference on Automatic Face \& Gesture Recognition. 2008

21. Gafurov D, Helkala K, Sondrol T. Biometric gait authentication using accelerometer sensor. Journal of Computers. 2006; 18:51C-59.

22. Gellersen HW, Schmidt A, Beigl M. Multi-sensor context-awareness in mobile devices and smart artifacts. Mob Netw Appl. 2002; 7(5):341-351.

23. Barbieri RH, Farella BE, Ricco BAL. A low-power motion capture system with integrated accelerometers gesture recognition applications. International Symposium on VLSI Technology, Systems and Applications. 2004

24. Hackmann G, Chipara O, Lu C. Robust topology control for indoor wireless sensor networks. ACM Conference on Embedded Network Sensor Systems. 2008:57-70. 
25. J L, Z W, L Z, Wickramasuriya, Vasudevan VJ. uwave: Accelerometerbased personalized gesture recognition and its applications. IEEE International Conference on Pervasive Computing and Communications. 2009

26. Kale A, Cuntoor N, Kruger V. Gait-based recognition of humans using continuous hmms. Fifth IEEE International Conference on Automatic Face and Gesture Recognition. 2002

27. Kleisouris K, Firner B, Howard R, Zhang Y, Martin RP. Detecting intra-room mobility with signal strength descriptors. MobiHoc '10. 2010:71-80.

28. Kunze, K.; Lukowicz, P. ISWC '07: Proceedings of the 2007 11th IEEE International Symposium on Wearable Computers. Washington, DC, USA: IEEE Computer Society; 2007. Using acceleration signatures from everyday activities for on-body device location; p. 1-2.

29. Kunze, K.; Lukowicz, P.; Junker, H.; Trster, G. LOCA04: International Workshop on Location and Context-Awareness. Springer-Verlag; 2005. Where am i: Recognizing on-body positions of wearable sensors; p. 264-275.

30. Lee J, Sutter K, Askew C. Symmetry in running gait using a single inertial sensor. Journal of Science and Medicine in Sport. 2010; 5:559-563. [PubMed: 19850518]

31. Lester J, Hannaford B, Borriello G. using accelerometers to determine if two devices are carried by the same person. Proceedings of Second International Conference on Pervasive Computing (Pervasive 2004). 2004:33-50.

32. Lester J, Hartung C, Pina L, Libby R, Borriello G, Duncan G. Validated caloric expenditure estimation using a single body-worn sensor. UBICOMP '09. 2009:225-234.

33. Link JAB, Fabritius G, Alizai MH, Wehrle K. Burrowview: Seeing the world through the eyes of rats. the 2nd IEEE International Workshop on Information Quality and Quality of Service for Pervasive Computing. 2010

34. Mantyjarvi J, Kela J, Korpipaa P, Kallin S. Enabling fast and effortless customisation in accelerometer based gesture interaction. the 3rd international conference on Mobile and ubiquitous multimedia. 2004

35. Mayagoitia R, Lotters J, Veltink P, Hermens H. Standing balance evaluation using a triaxial accelerometer. Gait \& Posture. 2002; 16:55-59. [PubMed: 12127187]

36. Miluzzo E, Zheng X, Fodor K, Campbell AT. Radio characterization of 802.15.4 and its impact on the design of mobile sensor networks. Proc 5th European Conf on Wireless Sensor Networks (EWSN 08). 2008:171-188.

37. Minnen D, et al. Improving activity discovery with automatic neighborhood estimation. IJCAI '07. 2007:2814-2819.

38. Parijat P, Lockhart T. Effects of quadriceps fatigue on the biomechanics of gait and slip propensity. Gait Posture. 2008; 28:568C-573. [PubMed: 18514522]

39. Park BS, Youn JI. Topographic measurement of skin color by narrow-band reflectance spectrophotometer and minimal erythema dose in koreans. Skin Research and Technology. 1998; 4:14-17.

40. Shah RC, et al. On the performance of bluetooth and ieee 802.15.4 radios in a body area network. BODYNETS '08. 2008:1-9.

41. Vahdatpour A, Amini N, Sarrafzadeh M. Toward unsupervised activity discovery using multidimensional motif detection in time series. IJCAI '09. 2009:1261-1266.

42. Vahdatpour A, Amini N, Sarrafzadeh M. On-body device localization for health and medical monitoring applications. PerCom '11. 2011

43. Vahdatpour A, Sarrafzadeh M. Unsupervised discovery of abnormal activity occurrences in multidimensional time series, with applications in wearable systems. SDM '10: Siam Conference on Data Mining. 2010

44. Wegmueller MS, Kuhn JFA, Oberle M, Felber N, Kuster N, Fichtner W. An attempt to model the human body as a communication channel. IEEE Trans Biomedical Engineering. 2007; 54:18511857.

45. Weiss GM, Lockhart JW. Identifying user traits by mining smart phone accelerometer data. the Fifth International Workshop on Knowledge Discovery from Sensor Data (at KDD-11). 2011 
46. Welk G, Differding J. The utility of the digi-walker step counter to assess daily physical activity patterns. Medicine and Science in Sports and Exercise. 2000; 32(9):S481-S488. [PubMed: 10993418]

47. Xu R, Zhu H, Yuan J. Electric-field intrabody communication channel modeling with finite-element method. IEEE Trans Biomedical Engineering. 2011; 58:705-712. 


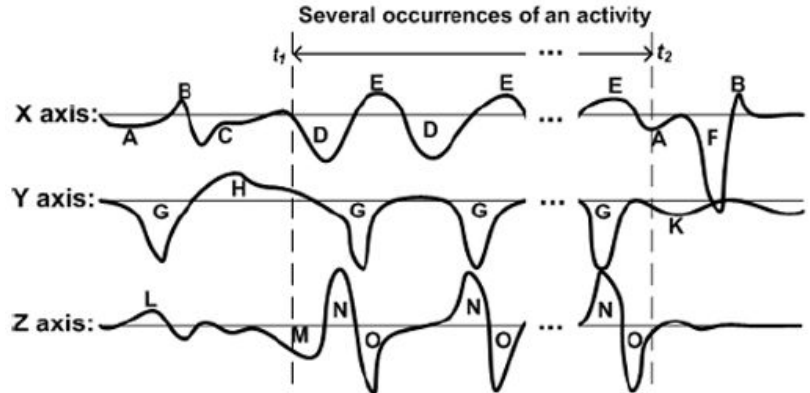

a)

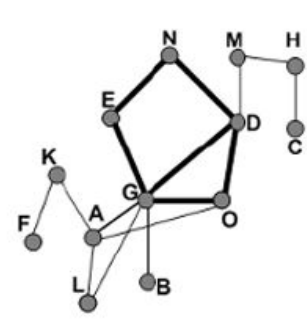

b)

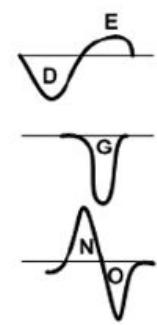

c)

Figure 1.

An illustrating example of the clustering algorithm for the recurrent activity discovery; a) three dimensions of accelerometer data, with several occurrences of an activity between $t_{1}$ and $t_{2}$. b) The interval coincidence graph, representing the primitives in the time series data. The thickness of edges show higher coincidence between the primitives. c) Primitives with high coincidence are clustered and the recurrent activity is discovered. $t_{1}$ and $t_{2}$ are sent to output as the start and end time of the most frequent activity. 


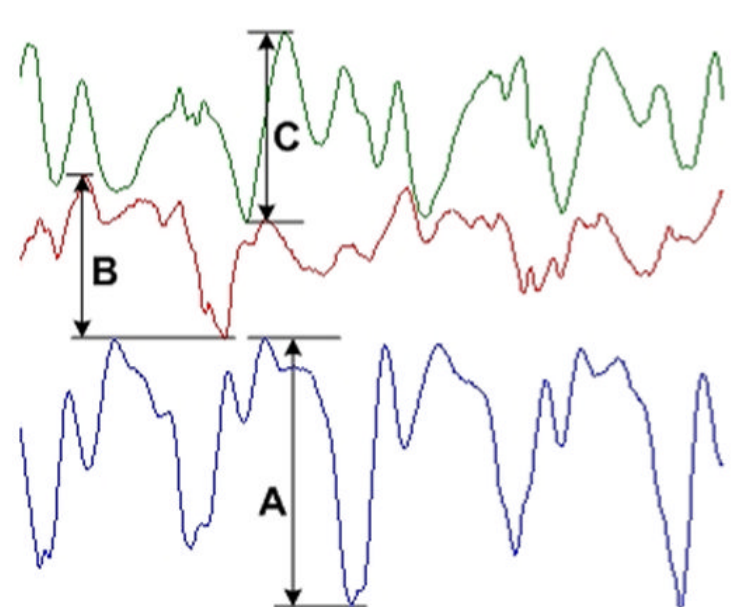

a)

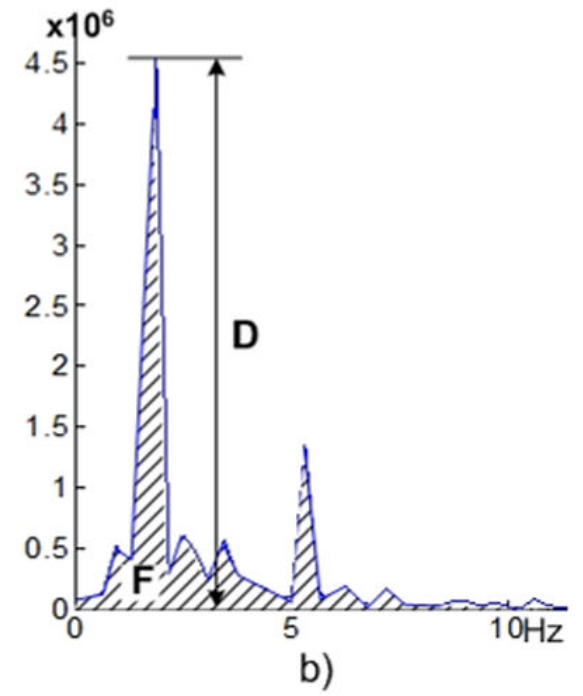

Figure 2.

a) Accelerometer time series captured during walking in a sensor mounted on the forearm (3 second interval). b) Power spectrum. 


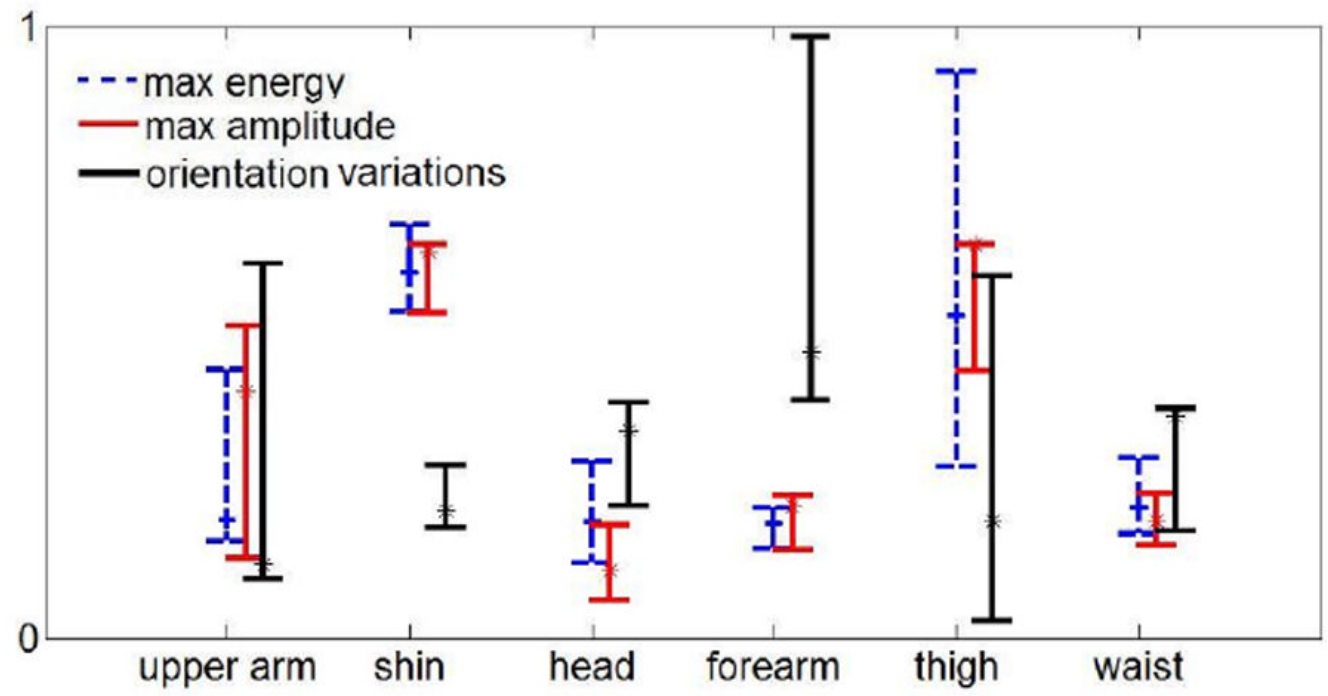

Figure 3.

Variation of three features for a subject in different sensor locations. Data is captured in a 5 minute time frame. $\mathrm{Y}$ axis is normalized. Blue is the maximum energy of the power spectrum, red is the maximum amplitude among all direction, and black is the average number of orientation variations in a 5 minute time frame. 


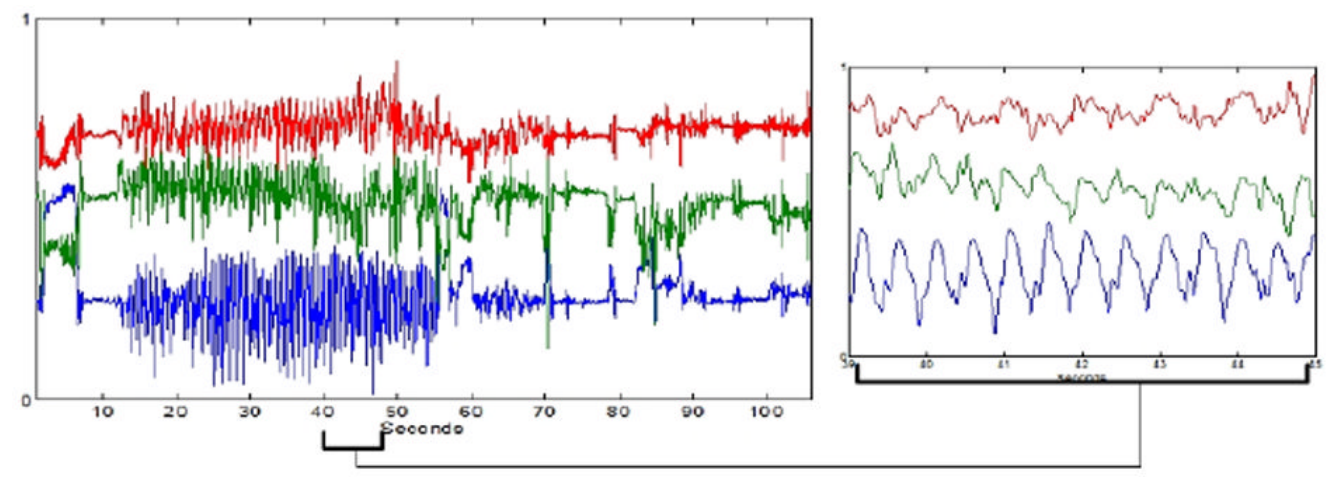

Figure 4.

Acceleration data collected from a sensor positioned on the arm during a 105 second interval (left). The magnified interval (right) depicts several occurrences of a walking activity. Y axis is normalized and $\mathrm{X}, \mathrm{Y}$, and $\mathrm{Z}$ acceleration dimensions are shifted to enhance the presentation. 


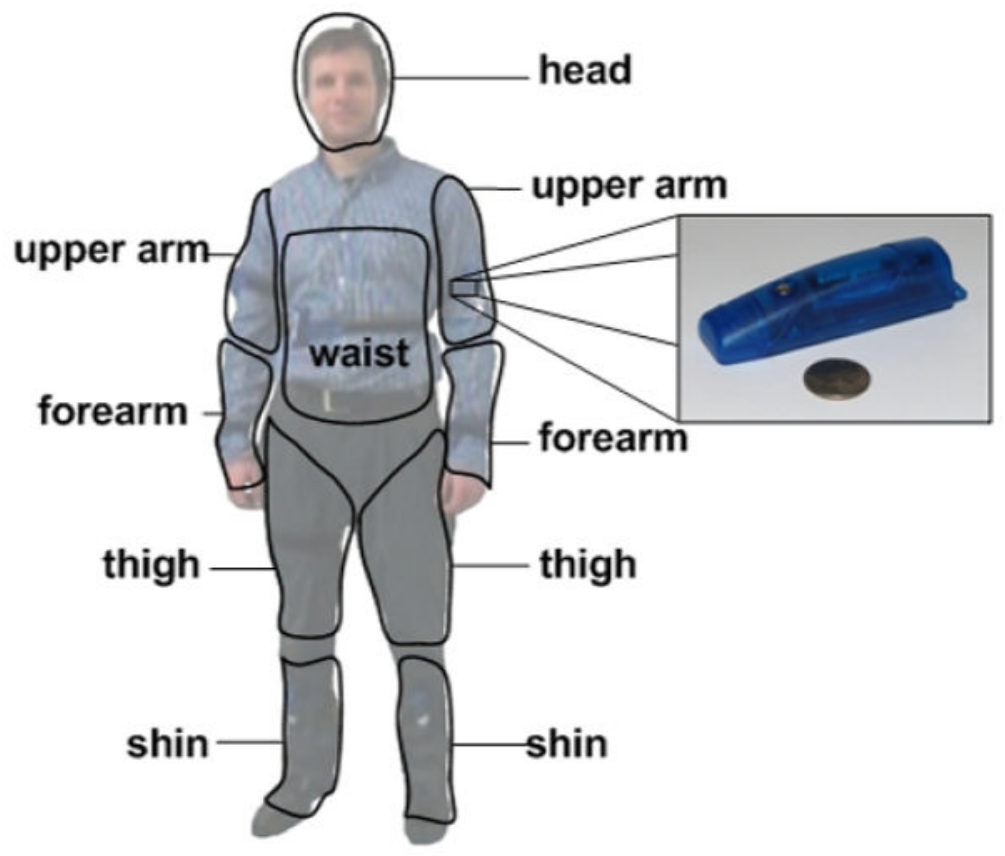

Figure 5.

Sensors are placed on 10 different regions on the body. The localization algorithm has 6 different outputs. \{forearm, upper arm, head, thigh, shin, waist . 


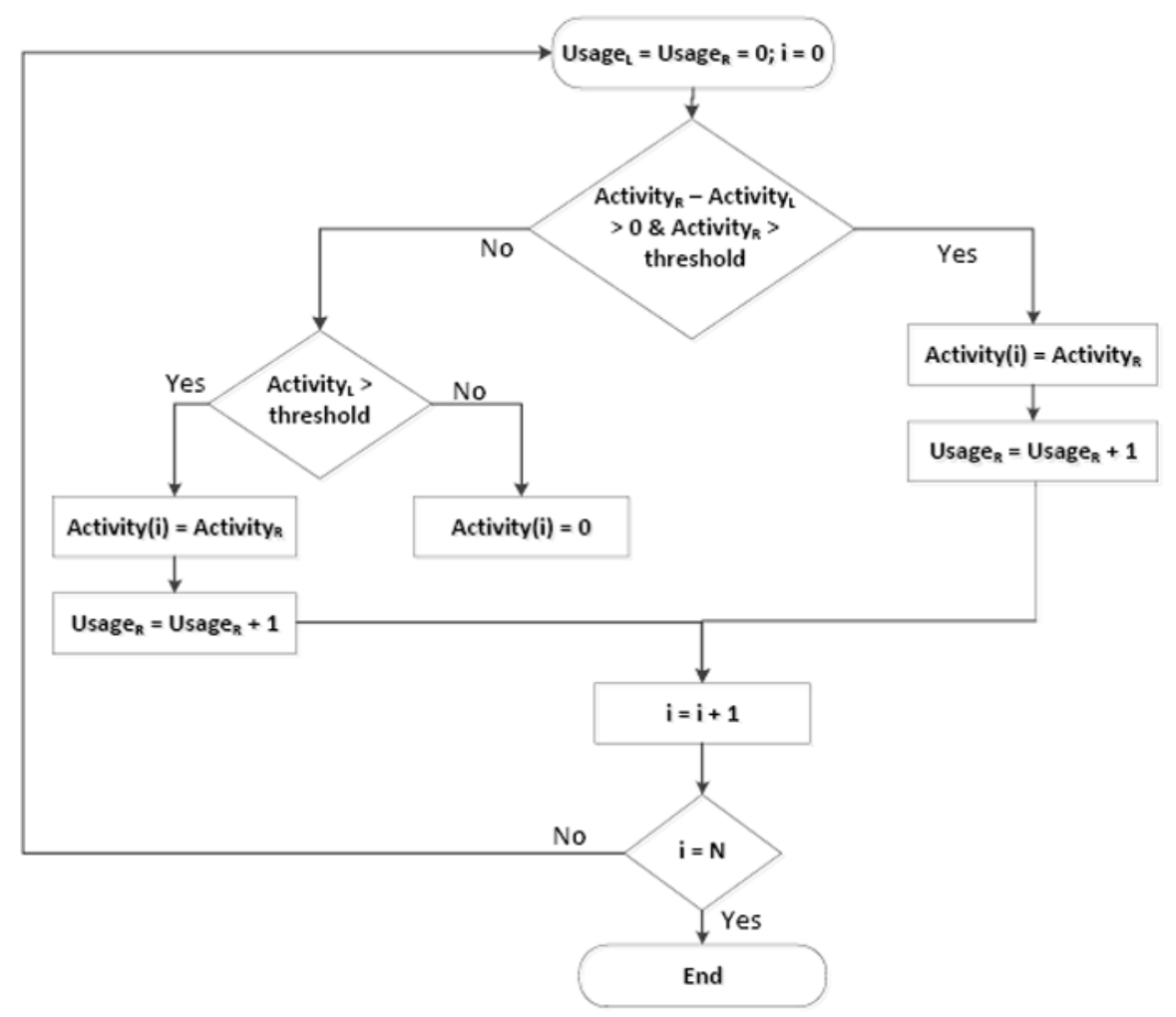

Figure 6.

Flowchart for estimating the asymmetry between left and right arms 


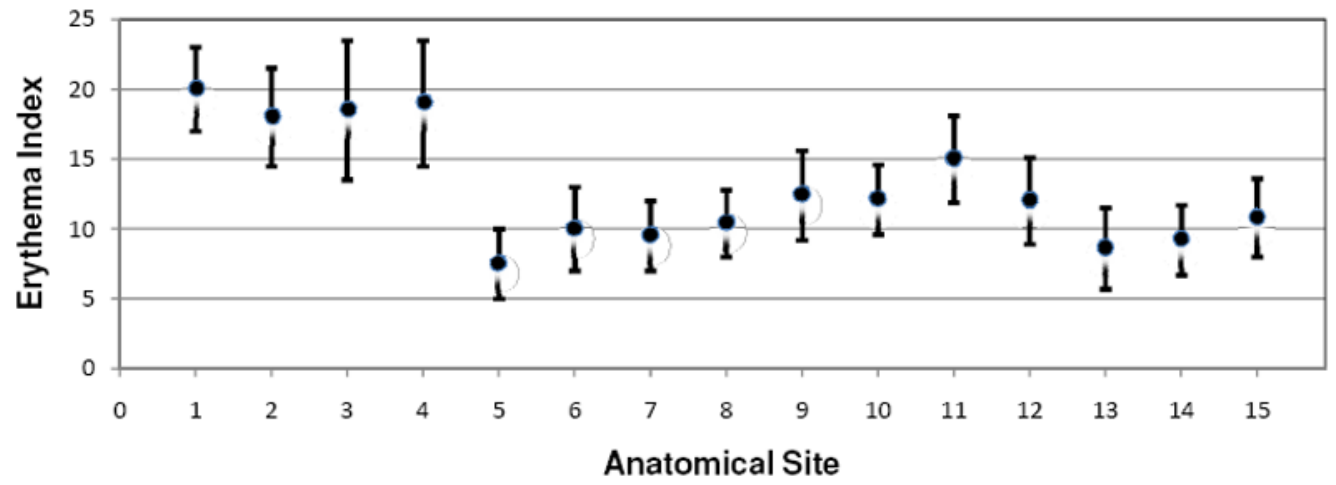

Figure 7.

Erythema index on different anatomical sites [39] 


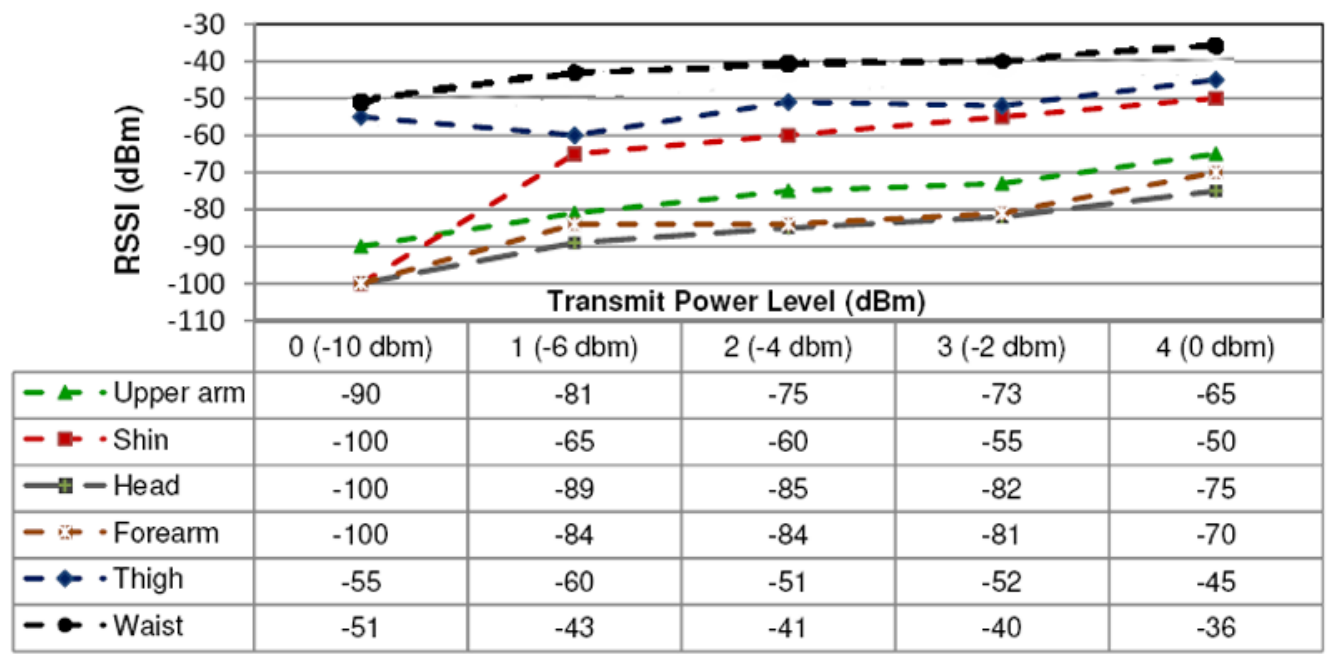

Figure 8.

Average RSSI measurements for different body parts at different transmission power levels 


\section{Table 1}

Normal temperature readings for different regions on the body [31]

\begin{tabular}{ccccc}
\hline Location & Ear & Axilla & Rectal & Oral \\
\hline Range $\left({ }^{\circ} \mathrm{C}\right)$ & $35.5-38$ & $35.5-37$ & $34.5-38$ & $33-38$ \\
\hline
\end{tabular}




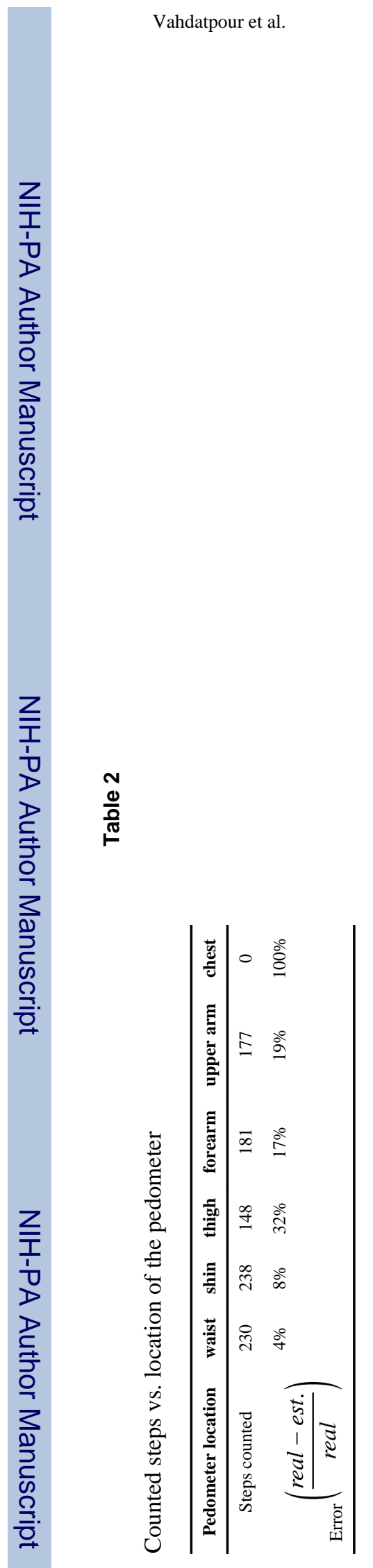

Page 28

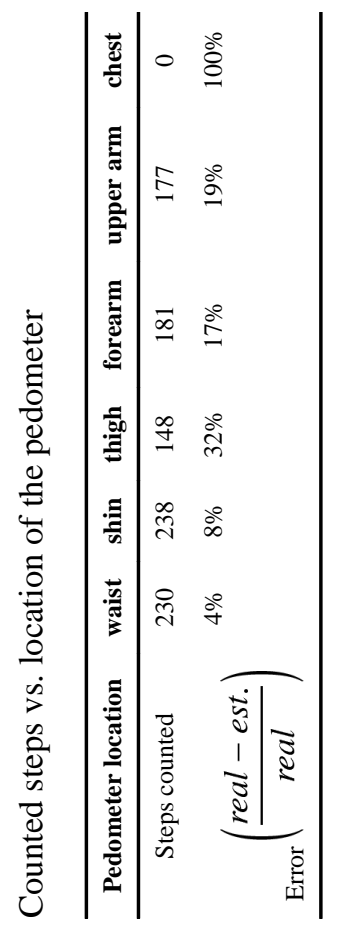

Pervasive Mob Comput. Author manuscript; available in PMC 2012 December 1. 
Table 3

The intuition behind the six major extracted features. Letters in parenthesis represent the features in Figure 2

\begin{tabular}{|l|l|l|}
\hline Activity & Feature & Characteristic \\
\hline Walking & Max energy in power spectrum (D) & $\begin{array}{l}\text { Impact of strides } \\
\text { on acceleration }\end{array}$ \\
\hline Walking & Sum of energy in power spectrum (F) & Motion range \\
\hline Walking & Maximum of Max amplitudes for all axes $(A>B, A>C)$ & $\begin{array}{l}\text { Degree of freedom } \\
\text { in movement }\end{array}$ \\
\hline Walking & Ratio of amplitude in different axes $\left(\frac{A}{C}, \frac{A}{B}\right)$ & \\
\hline Non-walk & Number of orientation variations & \multicolumn{1}{|c}{} \\
\hline
\end{tabular}




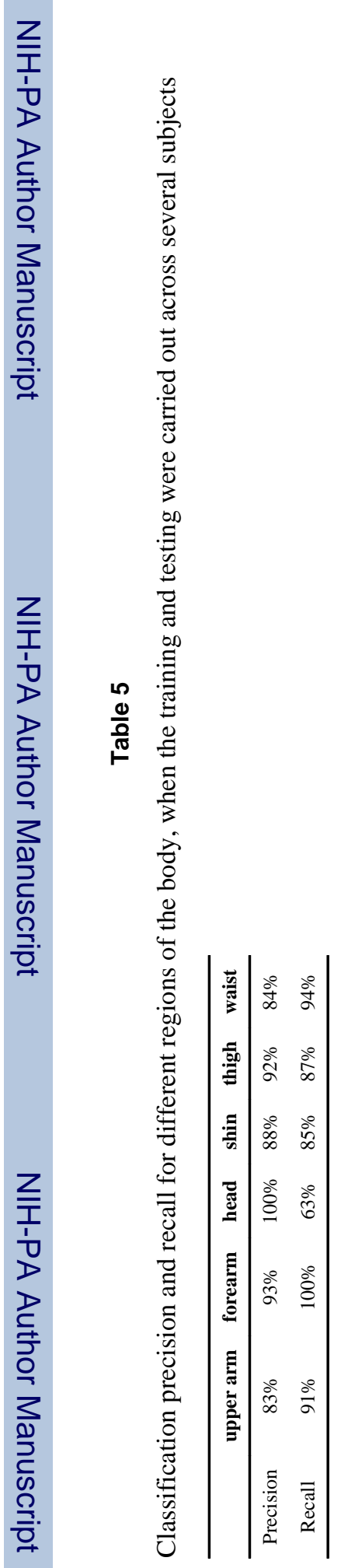

Pervasive Mob Comput. Author manuscript; available in PMC 2012 December 1. 
Table 6

Right arm usage and left arm usage while walking for 100 gait cycles

\begin{tabular}{|c|c|c|}
\hline Subject & Left Arm Usage (\%) & Right Arm Usage (\%) \\
\hline 1 & 29 & 71 \\
\hline 2 & 62 & 38 \\
\hline 3 & 57 & 43 \\
\hline 4 & 46 & 54 \\
\hline 5 & 66 & 34 \\
\hline 6 & 55 & 45 \\
\hline 7 & 71 & 29 \\
\hline 8 & 36 & 54 \\
\hline 9 & 59 & 41 \\
\hline 10 & 65 & 35 \\
\hline 11 & 31 & 69 \\
\hline 12 & 46 & 54 \\
\hline 13 & 49 & 51 \\
\hline 14 & 45 & 55 \\
\hline 15 & 41 & 59 \\
\hline 16 & 57 & 43 \\
\hline 17 & 47 & 53 \\
\hline 18 & 43 & 57 \\
\hline 19 & 41 & 59 \\
\hline 20 & 42 & 58 \\
\hline 21 & 56 & 44 \\
\hline 22 & 59 & 41 \\
\hline 23 & 42 & 58 \\
\hline 24 & 60 & 40 \\
\hline 25 & 41 & 59 \\
\hline Mean & 49.84 & 50.16 \\
\hline$\sigma$ (S.D.) & 10.55 & 11.13 \\
\hline
\end{tabular}




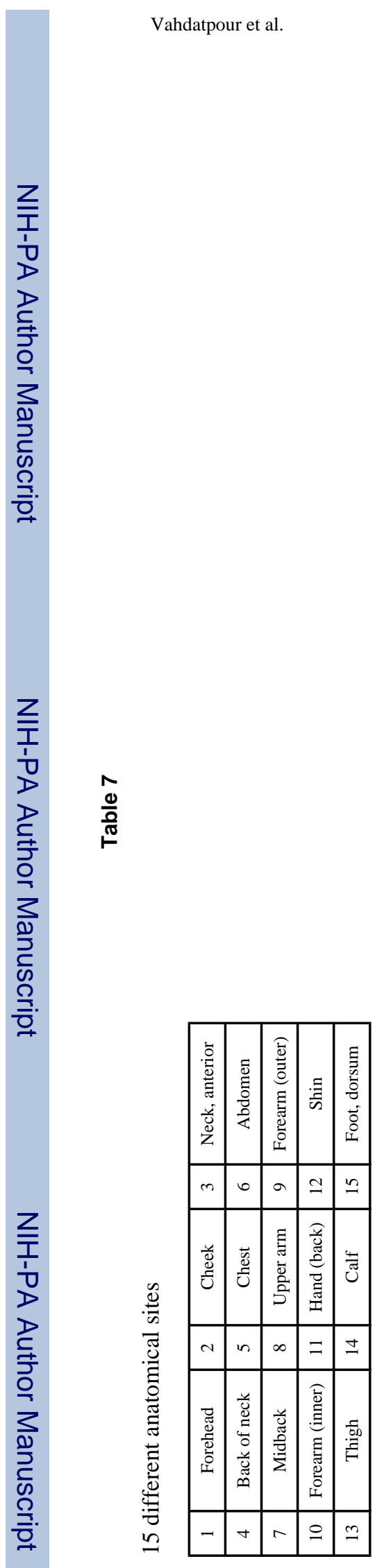

Pervasive Mob Comput. Author manuscript; available in PMC 2012 December 1. 
Table 8

Comparison of fraction of UV that each anatomical site receives

\begin{tabular}{|c|c|c|}
\hline Anatomical site & Fraction of total radiation on the first subject's body & Fraction of total radiation on the second subject's body \\
\hline Forehead & 0.33 & 0.29 \\
\hline Cheek & 0.30 & 0.32 \\
\hline Neck, anterior & 0.37 & 0.41 \\
\hline Back of neck & 0.45 & 0.40 \\
\hline Abdomen & 0.51 & 0.50 \\
\hline Midback & 0.46 & 0.44 \\
\hline Upper arm & 0.70 & 0.65 \\
\hline Forearm (outer) & 0.61 & 0.55 \\
\hline Forearm (inner) & 0.33 & 0.25 \\
\hline Hand (back) & 0.22 & 0.30 \\
\hline Shin & 0.32 & 0.37 \\
\hline Thigh & 0.37 & 0.29 \\
\hline Calf & 0.34 & 0.28 \\
\hline Foot (dorsum) & 0.22 & 0.30 \\
\hline
\end{tabular}


Table 9

Experiment setup

\begin{tabular}{|c|c|c|}
\hline Experiment Configuration & Transmitter & Receiver \\
\hline Address & 1 & 2 \\
\hline PAN ID & 70 & 70 \\
\hline Channel & 11 & 11 \\
\hline Destination Address & 2 & 1 \\
\hline Payload & 30 bytes & N/A \\
\hline ACKs & No & No \\
\hline Receiver's sensitivity & N/A & $-92 \mathrm{dBm}$ \\
\hline Modulation & OQPSK & OSPSK \\
\hline Transmit/Receiver current & $45 \mathrm{~mA}$ at $0 \mathrm{dBm}$ & $50 \mathrm{~mA}$ \\
\hline
\end{tabular}

